\title{
Softening of the actin cytoskeleton by inhibition of myosin II
}

\author{
Jan Christian Martens • Manfred Radmacher
}

Received: 15 October 2007 / Accepted: 5 December 2007 /Published online: 30 January 2008

(C) Springer-Verlag 2008

\begin{abstract}
We have investigated the mechanical properties of fibroblast cells after adding the myosin inhibitor blebbistatin and the Rho-kinase inhibitor Y-27632 by atomic force microscopy (AFM). We have observed a decrease in the elastic modulus from a value of around $20 \mathrm{kPa}$ down to a value around $8 \mathrm{kPa}$ on a time scale of around 30-60 min when applying the myosin inhibitor blebbistatin, whereas the Y-27632 did not show any prominent mechanical effects. From topographic images, we can conclude that, after adding blebbistatin, actin filaments are not visible any more, whereas Y-27632 did not show any prominent effects in cell morphology. This study shows that tension generated by myosin contributes to the cellular stiffness and thus can be observed by measuring the elastic modulus of cells.
\end{abstract}

Keywords Actin · Cytoskeleton · Mechanical properties . Myosin · Fibroblast

\section{Introduction}

The actin cytoskeleton of eukaryotic cells is responsible for cell morphology, but also for many cellular processes including cell migration and cell division. Changes in the architecture and structure of the cytoskeleton, mediated by a large number of actin binding molecules, can result in changes in mechanical properties of this polymeric network. Thus, it has been proposed by Elson et al. to use cellular mechanics as a reporter of cellular processes [3],

J. C. Martens $\cdot$ M. Radmacher $(\bowtie)$

Institut für Biophysik, Universität Bremen,

Bremen, Germany

e-mail: mr@biophysik.uni-bremen.de which has been implemented first by cell poking [2]. Especially atomic force microscopy (AFM) has been proven to be very helpful in investigating the mechanical response of cells with very high spatial resolution [17]. When taking force curves while scanning the sample (force mapping) [18], the elastic properties of cells can be probed locally [19]. Especially when studying cellular processes, AFM can help to give new insights into cellular behavior. Examples are the study of the mechanics of cytokinesis [11] and cell migration $[15,21]$. It has been shown that disruption of actin filaments results in a softening of cells [22].

The mechanical properties of cells are also dependent on cytoskeletal tension generated by myosin, which is important in many cellular processes, e.g., for cell morphology and shape by an interplay of local adhesion (focal adhesion points) and tension between these adhesion points. Cellular tension is mainly produced by non-muscle myosin II, which crosslinks actin filaments and thus can contract the actin cytoskeleton. There are several biochemical pathways to affect the activity of myosin. Blebbistatin directly inhibits myosin function very specifically [8] and has been used for instance - to study the function of myosin in cytokinesis [27]. Other drugs that affect the biochemical pathways activating myosin are ML-7 [23], which is an inhibitor of MLCK (myosin light chain kinase), and Y-27632 [10], which inhibits Rho-kinase, which also activates myosin. These drugs have been characterized well in biochemical activity assays or biophysical migration assays [5, 13, 13]; however, mechanical data have only been obtained in a few cases [10], including previous work by AFM, where the influence of ML-7 on cellular stiffness has been investigated [25]. In this study, a decrease of Young's modulus by a factor of three has been observed in NRK fibroblast cells. These studies [29] suggest that the two different biochemical pathways activating myosin (via ROCK or via MLCK) 
are regulated differently, depending on cell type, cell activity, and the cellular process investigated.

\section{Materials and methods}

Cell culture

NRK cells were grown in Dulbecco's modified Eagle's medium (DMEM, Biochrom, Berlin, Germany) with supplemented $10 \%$ fetal calf serum (FCS, Biochrom), penicillin and streptomycin (1\%), and $1 \%$ non-essential-amino acids (NEA). Cells were cultured at $37^{\circ} \mathrm{C}$ at $5 \% \mathrm{CO}_{2}$. Cells were cultured in plastic Petri dishes (Nunclon 947039 \& 9407395, Rochester, NY, USA) and plated 1 day before the AFM experiments.

Atomic force microscopy (AFM)

An AFM (MFP3D, Asylum-Research, Santa Barbara, CA, USA) was used to measure the topography and mechanical properties of cells. The AFM was combined with an optical microscope (Axiovert 200, Zeiss, Oberkochen, Germany) to be able to control the position of the AFM tip. We used very soft cantilevers (MLCT, VEECO) with a spring-constant of $0.01 \mathrm{Nm}^{-1}$. The Petri dishes were mounted in a homebuilt aluminum holder with vacuum grease, which was fixed by magnets to the base of the AFM. The AFM was put in a polymethylmethacrylate (PMMA) box to maintain $5 \% \mathrm{CO}_{2}$. The temperature of the sample was not controlled and was usually around $25^{\circ} \mathrm{C}$. The entire microscope was placed on a granite plate suspended with rubber bands from the ceiling to isolate it from vibrations.

\section{Phase-contrast and video microscopy}

Long-time control experiments were done by video light microscopy using phase contrast. (Axiovert 135 TV, Zeiss; Time-Lapse-Videorecorder: TL700, Panasonic, Osaka, Japan). A PMMA box was added to the microscope to maintain 5\% $\mathrm{CO}_{2}$. Therefore, observations over several hours of live cells were possible. Hence, the effect of the drugs used on cell morphology and the recovery from these drugs could be observed.

\section{Chemicals}

We dissolved Rock-Inhibitor (Y-27632, (+)-(R)-trans-4-(1aminoethyl)-N-(4-pyridyl)cyclohexanecarboxamide dihydrochlorate) in water at a concentration of $1.04 \mathrm{mM}$. Blebbistatin (1-phenyl-1,2,3,4-tetrahydro-4-hydroxpyrrolo[2,3-b]-7-methylquinolin-4-one) was dissolved in DMSO at a concentration of $3.05 \mathrm{mM}$. Fifty microliters of either stock solution was added to the medium to the Petri dishes $(1 \mathrm{ml})$, which resulted in a final concentration of $17 \mu \mathrm{M}$ for Y-27632 and $50 \mu \mathrm{m}$ for blebbistatin. Drugs were stored in a refrigerator and removed only right before adding them to the Petri dishes because especially blebbistatin is known to be light-sensitive [24].

Data analysis

Force curve data were analyzed to obtain elastic moduli as described elsewhere [16]. Briefly, we used Sneddon's modification [26] of the Hertzian model [4, 6] for the elastic response of a soft material indented by a conical punch. In this case, the indentation and loading force are related as follows:

$F(\delta)=\frac{\pi}{2} \frac{E}{1-v^{2}} \delta^{2} \tan \alpha$

Data analysis has been performed using home-built software written in IGOR (Wavemetrics, Lake Oswego, OR, USA). Loading forces were up to $800 \mathrm{pN}$; however, the range of data analyzed corresponded to loading forces between 100 and $400 \mathrm{pN}$ with typical indentations of 100 $200 \mathrm{~nm}$.

\section{Results and discussion}

We have evaluated the response to the drugs used here in long-time video phase contrast microscopy over several hours to verify the activity of our drugs and to find a concentration regime where morphological changes could be picked up in most cells. Cell recovery has also been observed after a time period of $1-2 \mathrm{~h}$ (data not shown here). In addition, phase contrast microscopy showed similar morphological changes due to the application of drugs, as has been observed in the AFM. Thus, we can conclude that morphological changes in the AFM are not due to the application of mechanical force but due to the drugs applied. These video microscopy studies also confirmed the activity of our drugs, e.g., the activity of blebbistatin has not been modified by the illumination conditions in our setup, although it is known to be light-sensitive and its activity is decreased by light of a wavelength of $488 \mathrm{~nm}$ [24]. Because the laser diode of the AFM emitted light in the near infrared, we do not expect any drastic reduction of blebbistatin activity either.

Figure 1 presents contact mode AFM images of NRK fibroblast cells before and after adding drugs. In AFM contact mode images, single actin filaments cannot be resolved, so prominent features are actin filament bundles [22], which are clearly visible in images of fibroblast cells here and have been observed in many other cell types. In the case of blebbistatin, a retraction of the cell is observed 
Fig. 1 Typical AFM images of NRK fibroblasts before and after adding blebbistatin and rock-inhibitor Y-27632. a An AFM image of the same cell, which is shown in $\mathbf{b}$ after adding blebbistatin. Blebbistatin results in a decrease of cytoskeletal tension. Actin filamentous structures disappear due to the cross-linking function of myosin, which is inhibited. Thus, cells are retracting slightly. c An AFM image of the same area of a cell, which is also shown in $\mathbf{d}$ after Y-27632 has been added. The Rho-kinase inhibitor Y-27632 does not have any obvious effect on cell morphology and the appearance of the actin cytoskeleton. Filamentous structures are visible before and after its application
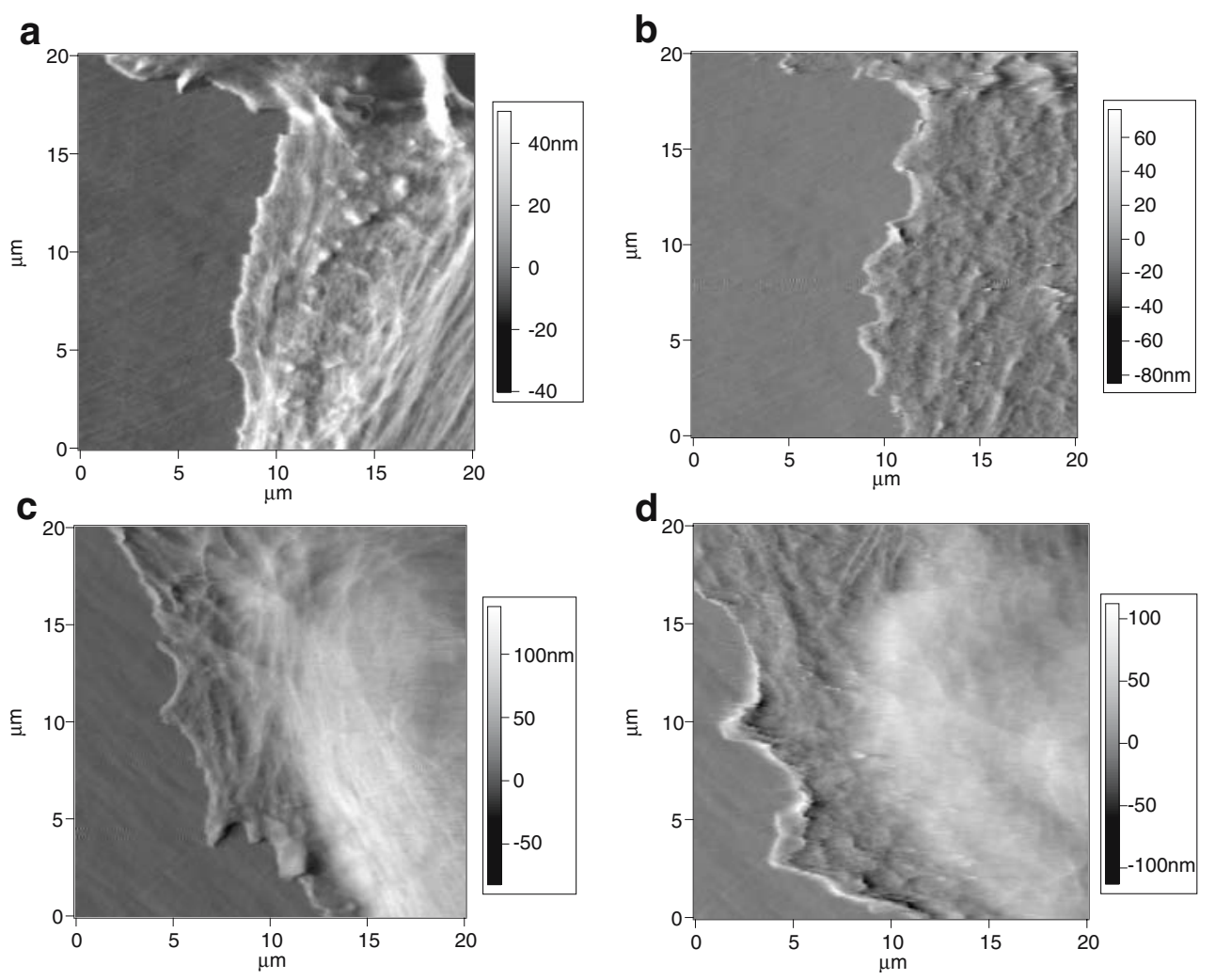

(Fig. 1b) and filamentous structures clearly visible in the untreated cells (Fig. 1a) disappear. In the case of Y-27632, no drastic effects can be observed. Cells do not drastically change their morphology as they usually do in life imaging, and filamenteous structures are visible before and after treatment, although it appears to be less fiber-like structures in the presence of the drug. It is known that blebbistatin reduces the activity of myosin, which cross-links actin filaments. In AFM topographic images, mainly actin filament bundles (stress fibers) are visible, whereas single filaments in a random actin network cannot be resolved. Y-27632 inhibits Rho-kinase, which can then lead to a decreased activity of myosin.

Figure 2 shows typical force curves on NRK cells before and after adding blebbistatin. We have verified first that the stiffness of cells stays constant over a time course of an hour and more if no drug is applied or if only DMSO is applied (see Fig. 5). The force curves have been fitted with the Hertz model to obtain elastic modulus values. In this case, we see a reduction from initial elasticity values of $25 \mathrm{kPa}$ by a factor of 3 . However, these data have to be interpreted with some caution. Cells are retracting, so taking two force curves on the same sample position will correspond to different locations on the cell. Because cells are retracting, it is likely that the sample thickness will change; in the case of retraction, it will become smaller in general. Because, in indentation data on thin samples, the underlying stiff substrate (plastic Petri dish) will influence the observed stiffness, elastic moduli will be over-estimated [1]. This typically occurs when the indentation becomes comparable to sample thickness in AFM indentation experiments. In cells, this is usually the case for thickness of and below $1 \mu \mathrm{m}$. Although there have been several attempts to include this thickness effect in an advanced or expanded Hertz model, none of these expansions are applicable in

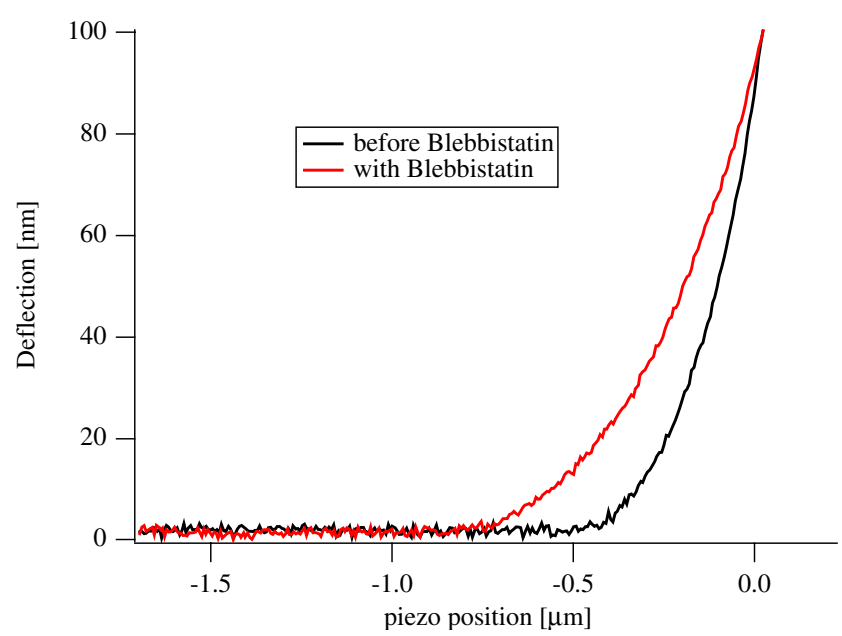

Fig. 2 Typical force curves before (lower trace) and after (upper trace) adding blebbistatin. The force curves were taken on the same cell at areas of comparable thickness. However, because the cells are changing morphology, they are not taken at the same position. The data are fitted with the Hertz model yielding Young's moduli of 25 and $8 \mathrm{kPa}$, respectively [9] 
general $[7,9,14,12,20]$. One issue is that an important parameter is the contact between cell and support, where the two limiting cases are slip or non-slip boundaries. The latter corresponds to a firm attachment of the cell to the substrate, whereas the former corresponds to a cell, which can freely slide along the substrate. Because cells exhibit areas with one or the other characteristic (focal adhesion points are firmly attached, the areas in between may be free to slide laterally), it is not clear which model to use. Therefore, we have chosen a different route to follow changes in elastic moduli: when focusing on data at a certain thickness, temporal changes in stiffness, e.g., due to the application of drugs, can be followed quantitatively. This can be done easily by analyzing force map data appropriately.
Figure 3 shows a typical elasticity map of a NRK cell. An array of $16 * 16$ force curves has been recorded and each single force curve has been analyzed using the Hertz model as described above. The elastic modulus has then been plotted as a false color image. A series of images has been recorded where blebbistatin has been added right after the first data set $(0 \mathrm{~min})$. In these elasticity maps, the stiff plastic Petri dishes appear in blue colors, whereas the cell exhibits elastic moduli between $20-40 \mathrm{kPa}$ (first images yellow and green) and at later stages around or below $10 \mathrm{kPa}$ (red colors). From the elasticity maps, the shape of the cell can also be discerned, which shows that the cell is retracting.

In retraction, the peripheral parts of cells usually become thinner, which could result in an artificial stiffening of the
Fig. 3 Elasticity map of a NRK cell before $(0 \mathrm{~min})$ and after adding blebbistatin to the medium (a-f). The cell is retracting and, at the same time, softening. Blue (large Young's modulus) corresponds to the substrate, whereas all other values of the elastic modulus correspond to the cell. The area covered by the cell is decreasing because the cell is retracting; at the same time, the cell is softening (gradual transition from high stiffness values around 20-40 $\mathrm{kPa}$ (yellow and green) to values around and below $10 \mathrm{kPa}($ red $)$ )
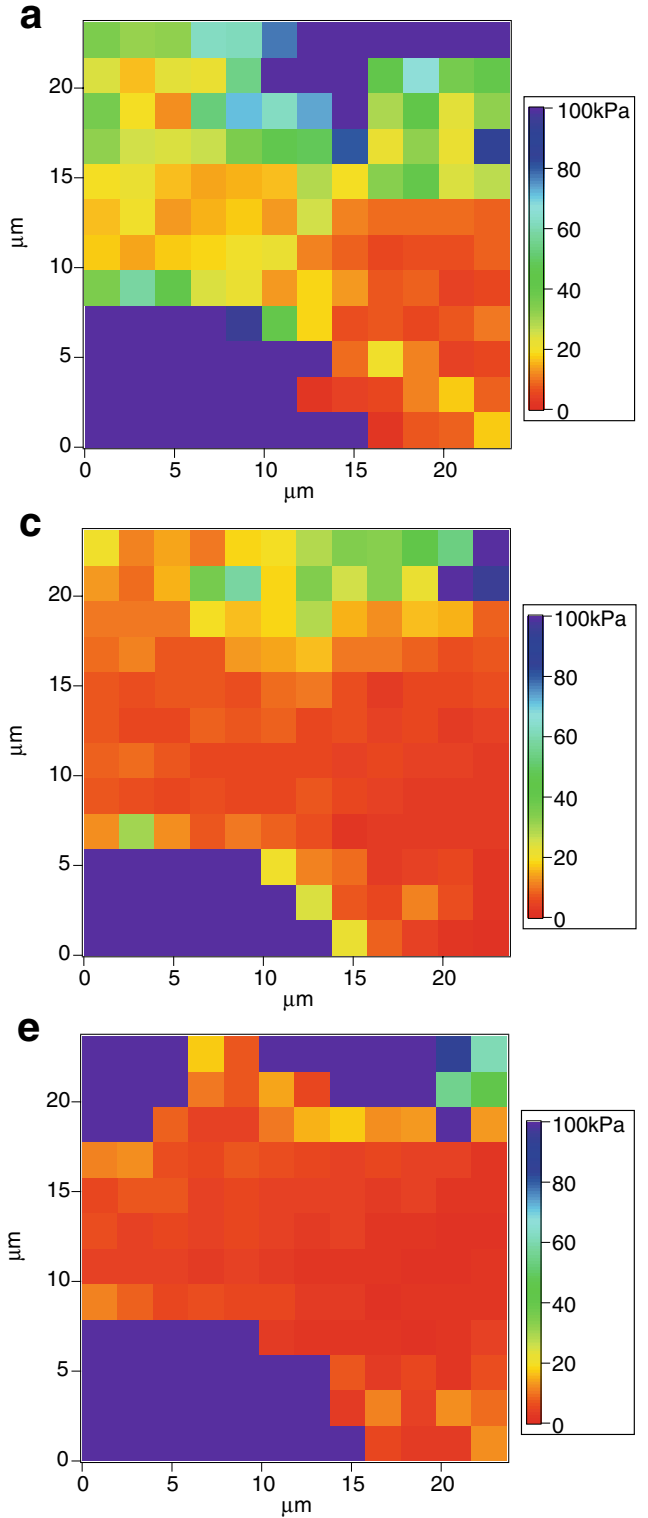
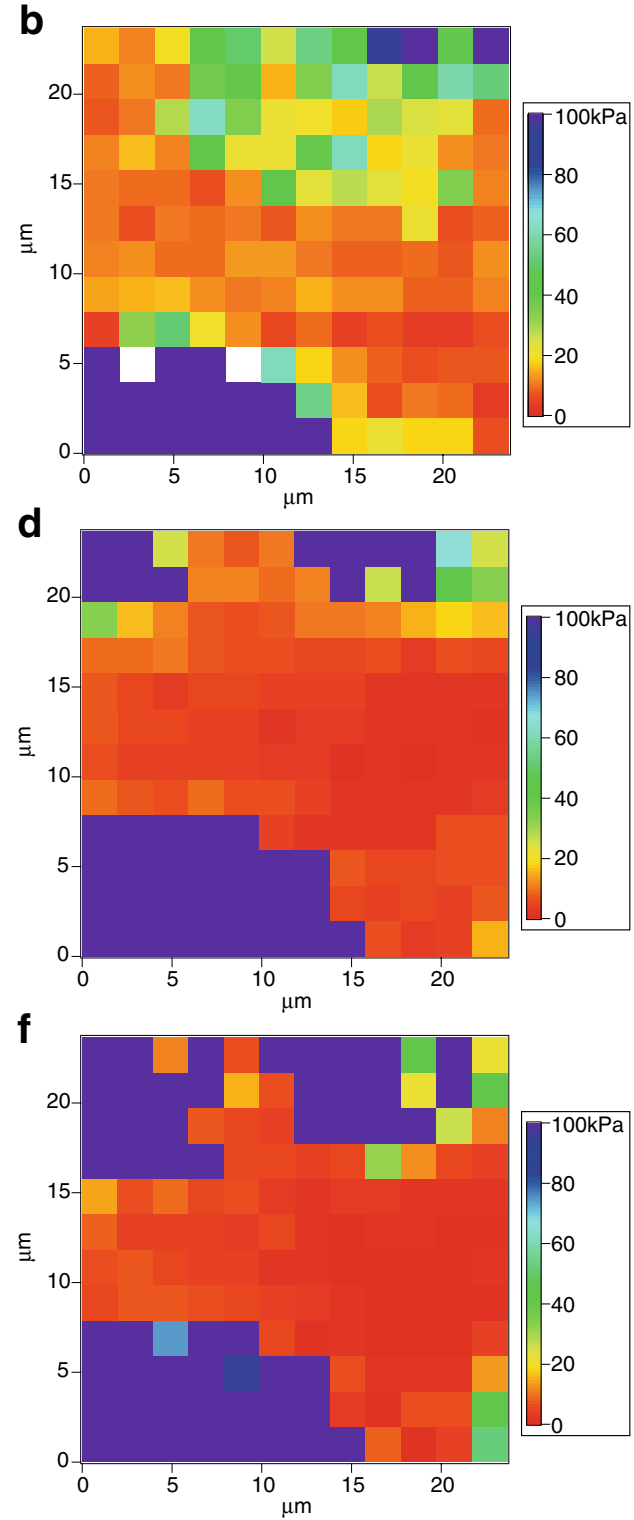
apparent stiffness. However, here, a softening occurs, which cannot be explained by this thickness artifact. However, to quantify the decrease in stiffness, it is best to compare only stiffness values of identical sample thickness, as has been introduced in a previous study [25]. Therefore, we have plotted the elastic modulus as a function of sample thickness (Fig. 4). When picking a typical thickness value, e.g., $1 \mu \mathrm{m}$, for each force map, temporal changes in stiffness regardless of topographical changes can be observed.

Figure 5 shows the time evolution of the (apparent) elastic modulus at a sample thickness of $1 \mu \mathrm{m}$ after adding blebbistatin for two typical experimental runs (different cells from different experiments). In both cases, a decrease of the elastic modulus by a factor of 3 has been observed.

It is very surprising that we have not observed any wellpronounced change in elastic properties after adding the Rho-kinase inhibitor Y-27632 to the medium, although its biochemical activity on myosin activity has been observed in several instances. We have occasionally observed a transient decrease in stiffness, by a factor of 3 to 4 ; however, this decrease did not last long enough to be measured repeatedly. This may be due to the limited time resolution in our force map measurements (typically $6 \mathrm{~min}$ ), so that in most cases, we miss these short deviations in stiffness. Another issue is that effects of Y-27632 may be too localized to be detectable. For example, a spatial difference between inhibition of MLCK and ROCK has been observed, especially in focal adhesions [28]. Therefore, the results on Y-27632 should probably be interpreted with some caution here.

The results presented here corroborate results from a previous study, where we have used different drugs (ML7 and KLM) to quantify their influence on cellular stiffness.

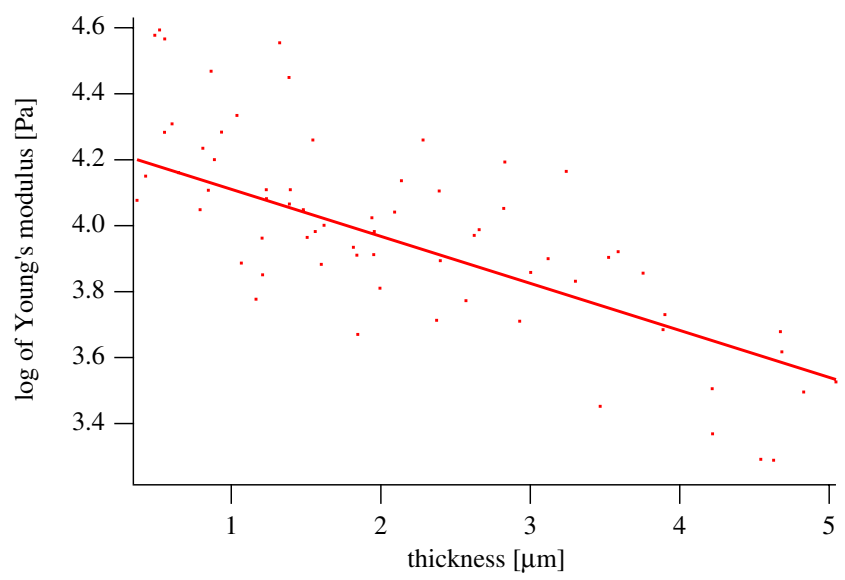

Fig. 4 Elastic modulus as a function of sample thickness. For sample thicknesses below $1 \mu \mathrm{m}$, the apparent stiffness will be affected by the thickness because the AFM tip "feels" the underlying stiff substrates. For larger thicknesses, the decrease in elastic modulus reflects real differences in architecture of the cytoskeleton. In the following, we compare areas of identical thickness to get comparable data

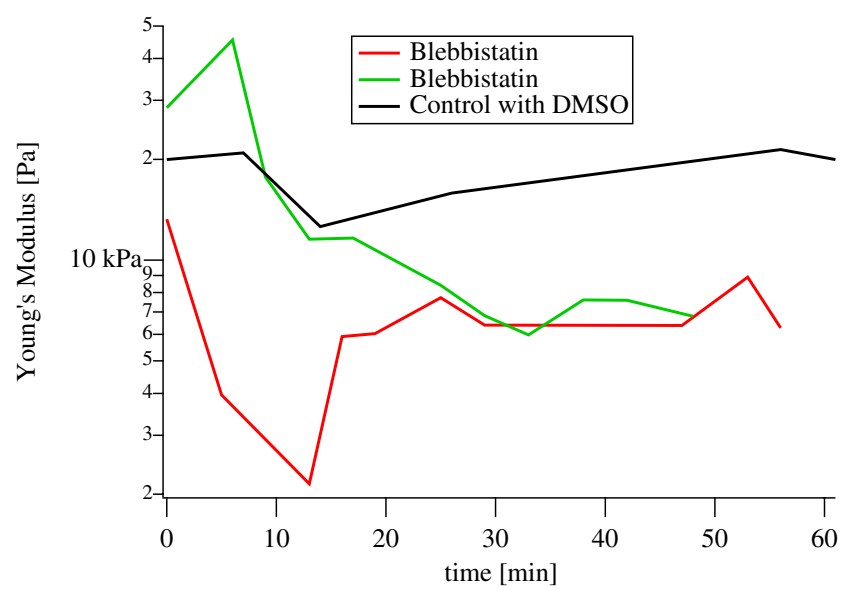

Fig. 5 Time evolution of the elastic modulus value for a given thickness $(1 \mu \mathrm{m})$ after adding blebbistatin to the medium for two different cells (red and green traces). The elastic modulus drops from initial values of $20 \mathrm{kPa}$ (top line) down to values around $8 \mathrm{kPa}$ over a time scale of $30 \mathrm{~min}$. Although cell 2 (green line) starts at a different elasticity value, it also becomes softer by a factor of 3 . Despite the variability in stiffness values, the cells behave similarly. The black trace shows a control measurement were only DMSO has been added. In this case, no change in elastic properties has been observed

Because the effects of the drugs used in the previous study are not entirely well understood, we picked two drugs here, where the effects have been characterized very well (blebbistatin) or - in comparison - where an influence via a biochemical pathway can be hypothesized (Y-27632). Because we have observed here a similar decrease in stiffness when adding blebbistatin, we can argue that the reduction in cellular stiffness, in all cases studied, is due to a decrease of myosin activity, resulting in a loss of cytoskeletal tension.

\section{Conclusions}

We have investigated the contribution of myosin II activity on the mechanical properties of cells as observed by indentation experiments on live cells by AFM. Blebbistatin, which inhibits myosin II directly, results in a retraction and softening of the cells. Y-27632, which inhibits Rho-kinase and, thus, can decrease myosin activity indirectly, did not show any detectable changes in stiffness. In contrast, in a previous study [25], inhibition of MLCK by ML-7 resulted in a decrease of stiffness as detected by indentation experiments by AFM. This suggests that myosin activity is regulated differently via MLCK or Rho-kinase dependent on cellular behavior. This difference in activation pathways, which has been found here in mechanical properties of cells, is in agreement with previous studies focusing on membrane protrusion and adhesion of fibroblasts by other methods [28]. 


\section{References}

1. Domke J, Radmacher M (1998) Measuring the elastic properties of thin polymer films with the AFM. Langmuir 14(12):3320-3325

2. Duszyk M, Schwab B III, Zahalak GI, Qian H, Elson EL (1989) Cell poking: quantitative analysis of indentation of thick viscoelastic layers. Biophys J 55:683-690

3. Elson EL (1988) Cellular mechanics as an indicator of cytoskeletal structure and function. Annu Rev Biophys Biophys Chem 17:397-430

4. Fung YC (1993) Biomechanics - mechanical properties of living tissues. Springer, Berlin Heidelberg New York

5. Guo W-h, Frey MT, Burnham NA, Wang Y-1 (2006) Substrate rigidity regulates the formation and maintenance of tissues. Biophys J 90:2213-2220

6. Hertz H (1882) Über die Berührung fester elastischer Körper. J Reine Angew Math 92:156-171

7. Kasas S, Wang X, Hirling H, Marsault R, Huni B, Yersin A, Regazzi R, Greeningloh G, Riederer B, Forrò L, Dietler G, Catsicas S (2005) Superficial and deep changes of cellular mechanical properties following cytoskeleton disassembly. Cell Motil Cytoskeleton 62:124-132

8. Limouze J, Straight A, Mitchison T, Sellers J (2004) Specificity of blebbistatin, an inhibitor of myosin II. J Muscle Res Cell Motil 25 (4):337-341

9. Mahaffy RE, Park S, Gerde E, Käs J, Shih CK (2004) Quantitative analysis of the viscoelastic properties of thin regions of fibroblasts using atomic force microscopy. Biophys J 86:1777-1793

10. Masayoshi U, Toshimasa I, Hiroyuki S, Takashi O, Toshio K, Tamami M, Hiroki T, Keiji Y, Jun I, Midori M, Shuh N (1997) Calcium sensitization of smooth muscle mediated by a Rhoassociated protein kinase in hypertension. Nature 389:990-994

11. Matzke R, Jacobson K, Radmacher M (2001) Direct, high resolution measurement of furrow stiffening during the division of adherent cells. Nat Cell Biol 3:607-610

12. McElfresh M, Baesu E, Balhorn R, Belak J, Allen MJ, Rudd RE (2002) Combining constitutive materials modeling with atomic force microscopy to understand the mechanical properties of living cells. Proc Natl Acad Sci U S A 99:6493-6497

13. Nakayama M, Amano M, Katsumi A, Kaneko T, Kawabata S, Takefuji M, Kaibuchi K (2005) Rho-kinase and myosin II activities are required for cell type and environment specific migration. Genes Cells 10(2):107-117

14. Ogilvy JA (1993) A parametric elastic model for indentation testing of thin films. J Phys D Appl Phys 26:2123-2131

15. Prass M, Jacobson K, Mogilner A, Radmacher M (2006) Direct measurement of the lamellipodial protrusive force in a migrating cell. J Cell Biol 174(6):767-772
16. Radmacher M (2007) Studying the mechanics of cellular processes by atomic force microscopy. In: Wang Y, Discher DE (eds) Cell mechanics. Academic, New York, pp 347-372

17. Radmacher M (2007) Studying the mechanics of cellular processes by atomic force microscopy. Methods Cell Biol 83:347-372

18. Radmacher M, Cleveland JP, Fritz M, Hansma HG, Hansma PK (1994) Mapping interaction forces with the atomic force microscope. Biophys J 66(6):2159-2165

19. Radmacher M, Fritz M, Kacher CM, Cleveland JP, Hansma PK (1996) Measuring the elastic properties of human platelets with the atomic force microscope. Biophys J 70(1):556-567

20. Rico F, Roca-Cusachs P, Gavara N, Farrè R, Rotger M, Navajas D (2005) Probing mechanical properties of living cells by atomic force microscopy with blunted pyramidal cantilever tips. Phys Rev E 72:021914

21. Rotsch C, Jacobson K, Radmacher M (1999) Dimensional and mechanical dynamics of active and stable edges in motile fibroblasts investigated by atomic force microscopy. Proc Natl Acad Sci U S A 96:921-926

22. Rotsch C, Radmacher M (2000) Drug-induced changes of cytoskeletal structure and mechanics in fibroblasts - an atomic force microscopy study. Biophys J 78:520-535

23. Saitoh M, Ishikawa T, Matsushima S, Naka M, Hidaka H (1987) Selective inhibition of catalytic activity of smooth muscle myosin light chain kinase. JBC 262(16):7796-7801

24. Sakamoto T, Limouze J, Combs CA, Straight AF, Sellers JR (2005) Blebbistatin, a myosin II inhibitor, is photoinactivated by blue light. Biochemistry 44(2):584-588

25. Schäfer A, Radmacher M (2005) Influence of myosin II activity on stiffness of fibroblast cells. Acta Biomater 1:273-280

26. Sneddon IN (1965) The relation between load and penetration in the axisymmetric Boussinesq problem for a punch of arbitrary profile. Int J Eng Sci 3:47-57

27. Straight A, Cheung A, Limouze J, Chen I, Westwood N, Sellers J, Mitchison T (2003) Dissecting temporal and spatial control of cytokinesis with a myosin II Inhibitor. Science 299(5613):17431747

28. Totsukawa G, Wu Y, Sasaki Y, Hartshorne DJ, Yamakita Y, Yamashiro S, Matsumura F (2004) Distinct roles of MLCK and ROCK in the regulation of membrane protrusins and focal adhesion dynamics during cell migration of fibroblasts. J Cell Biol 164(3):427-439

29. Totsukawa G, Yamakita Y, Yamashiro S, Hartshorne DJ, Sasaki Y, Matsumura F (2000) Distinct roles of ROCK (rho-kinase) and MLCK in spatial regulation of MLC phosphorylation for assembly of stress and focal adhesion in 3T3 fibroblasts. J Cell Biol 150:797-806 